\title{
Effects of Exposure to Cola-Based Soft Drink on Bleaching Effectiveness and Tooth Sensitivity of In-Office Bleaching: A Blind Clinical Trial
}

This article was published in the following Dove Press journal: Clinical, Cosmetic and Investigational Dentistry

\section{Viviane Hass $\mathbb{D}^{\prime}$ \\ Stephanye Tavares Carvalhal ${ }^{2}$ \\ Suellen Nogueira Linares \\ Lima $(\mathbb{D})^{2}$ \\ Andrés Alejandro Viteri-Garcia ${ }^{3}$ \\ Etevaldo Matos Maia Filho (D) ${ }^{2}$ \\ Matheus Coelho Bandeca $\mathbb{D I}^{2}$ \\ Alessandra Reis $\mathbb{D}^{4}$ \\ Alessandro Dourado \\ Loguercio (iD) ${ }^{4}$ \\ Rudys Rodolfo De Jesus \\ Tavarez $\mathbb{D D}^{2}$}

'Postgraduate Program in Dentistry, UNOPAR-University Northern Parana, Londrina, PR, Brazil; ${ }^{2}$ Postgraduate Program in Dentistry, University Ceuma, São Luis, Brazil; ${ }^{3}$ Escuela de la Salud Eugenio Espejo, Universidad UTE, Quito, Ecuador; ${ }^{4}$ Department of Restorative Dentistry, School of Dentistry, State University of Ponta Grossa, Ponta Grossa, Brazil
Correspondence: Alessandro Dourado Loguercio

Universidade Estadual de Ponta Grossa.

Departamento de Odontologia

Restauradora, Avenida Carlos Cavalcanti

4748 - Uvaranas, Ponta Grossa 84030-900,

PR, Brazil

Email aloguercio@hotmail.com
Objective: The purpose of this single-blind (evaluators) and parallel design study was to evaluate whether exposure to a cola-based soft drink during bleaching treatment with $35 \%$ hydrogen peroxide (HP) affects color change and bleaching-induced tooth sensitivity.

Material and methods: Forty-four patients with central incisors darker than A2 were selected. Participants who did not drink cola-based soft drinks were assigned to the control group (CG), while participants who drank a cola-based soft drink at least twice a day were assigned to the experimental group (EG). For the CG, foods with staining dyes were restricted. For the EG, there was no restriction on food and patients were asked to rinse their mouths with a cola-based soft drink for $30 \mathrm{~s}, 4$ times daily. For both groups, 2 sessions with three $15 \mathrm{~min}$ applications of $35 \%$ HP were performed. Shade evaluation was assessed via subjective (VITA classical and VITA bleacheguide shade guides) and objective methods (Easyshade spectrophotometer) at baseline, during bleaching (first, second, and third weeks), and post bleaching ( 1 week and 1 month). Patients recorded their sensitivity perceptions using a numerical rating scale and $0-10$ visual analog scales. Variation in shade guide units and the 2 colors (DE) were evaluated with a Student's $t$-test $(\alpha=0.05)$ and Mann-Whitney test $(\alpha=0.05)$. Absolute risk of tooth sensitivity and intensity of tooth sensitivity were evaluated by a Chi-square test $(\alpha=0.05)$.

Results: Effective bleaching was observed for both groups after 30 days, without statistical difference $(p>0.08)$. There was no significant difference in absolute risk of bleachinginduced tooth sensitivity between the 2 groups $(p=0.74)$. Higher and significant scores in pain scales were detected for the EG in comparison to the CG $(p<0.05)$.

Conclusion: Even that the cola-based soft drink exposure during in-office bleaching treatments did not affect the bleaching's effectiveness; patients reported a higher intensity in bleaching-induced tooth sensitivity.

Keywords: dentin sensitivity, dental bleaching, hydrogen peroxide, cola-based soft drink, carbonated beverages, cola, soda

\section{Introduction}

Dental bleaching is undoubtedly one of the most common clinical procedures in Dentistry probably because dental appearance is a determining factor in the attractiveness of a face and tooth color is considered the most important factor with regard to dental aesthetics. ${ }^{1}$ As it is an effective, conservative, and low-cost procedure, ${ }^{2}$ it should be considered the first choice for discolored teeth. ${ }^{3}$ When dealing with vital teeth, dental bleaching can be achieved through a variety of methods, such as in office 
(performed by the professional), at home (supervised by the professional), or both methods (combined or jump-start technique). ${ }^{4}$

Notwithstanding the effectiveness of the whitening technique, laboratory studies have reported alterations in enamel surface, such as an increase in roughness, demineralization potential, ${ }^{5,6}$ and permeability, ${ }^{7}$ consequently affecting the results of the bleaching treatment. Coloring and acidic $\mathrm{pH}$ agents, such as cola-based drinks, coffee, and wine, have been demonstrated to stain the dental structures and also affect the expected end result, as well as the longevity of the bleaching treatment. ${ }^{8-13}$

These findings support professionals' recommendations to their patients to avoid ingesting coloring agents in their diets during their bleaching treatments. ${ }^{14}$ However, when dealing with in vivo conditions, all dietary restrictions can be restricted, especially soft drinks.

Recently, a study ${ }^{15}$ revealed that bleaching agents, even in low concentrations, could result in changes in the dentinal collagen structure. Taking in consideration that dentin consists of a network of tubules, which transport fluids, ions, and molecules, it is unknown whether the changes could alter the retention of chromogenic molecules in dentin as well the sensitivity with intake coloring and acidic $\mathrm{pH}$ agents, as soft drink. One randomized clinical trial ${ }^{16}$ demonstrated that coffee consumption does not have an effect on color changes during a bleaching treatment. However, coffee solutions have a higher $\mathrm{pH}$ than cola-based soft drink. ${ }^{11,13}$ Observe that, several in vitro studies showed that colabased soft drinks are able to induce a similar or even higher color change than coffee during dental bleaching. However, to extent of author's knowledge, no clinical studies have been performed evaluated the effect of cola-based soft drink during bleaching treatment.

The cola-based soft drinks are widely intaken in Western world ${ }^{17,18}$ and several negative adverse effects are related to health indicators such especially the calcium homeostasis and bone turnover. ${ }^{19,20}$ In a Dentistry viewpoint, the intake of acidic beverages between meals, such as the cola-based soft drinks demonstrated a significative relation to erosion and, consequently with some degree of dentine hypersensitivity. ${ }^{21}$

It was to remember that, the success of bleaching treatment depends of the effectiveness of whitening as well the absence or reduced bleaching-induced tooth sensitivity, ${ }^{22,23}$ and considering the growing consumption soft drinks around the world, this clinical trial evaluated whether the effectiveness of bleaching, as well as tooth sensitivity, is affected by exposure to cola-based soft drinks during in-office bleaching treatments using 35\% hydrogen peroxide. The null hypotheses are that the exposure to cola-based soft drink during the bleaching treatment will not affect the (1) the effectiveness of bleaching, (2) the absolute risk of bleaching-induced tooth sensitivity and (3) the intensity of bleaching-induced tooth sensitivity.

\section{Materials And Methods}

This study was approved by the research ethics committee of the Ceuma University (UNICEUMA), according to protocol number. $1.422 .841(24 / 02 / 2016)$. It has also been registered in the registry of clinical trials Rebec under RBR-2nz5s2 number. All the volunteers signed a free informed consent form. This study was conducted in full accordance with the World Medical Association Declaration of Helsinki.

\section{Study Design, Setting, and Location of Data Collection}

This study was conducted in accordance with the Consolidated Standards of Reporting Trials statement. ${ }^{15}$ This was a singleblind (evaluators), parallel design with an equal allocation rate between groups, and it took place within the dental clinics of Local University from January 2017 to September 2017.

\section{Recruitment}

The participants who took part in this study were patients seeking dental treatment at the dental clinics at the university. A total of 58 participants were examined in a dental chair after dental prophylaxis with pumice and water to check whether they met the study's eligibility criteria.

\section{Eligibility Criteria}

Patients included in this clinical trial were between 18 and 40 years old and had good general and oral health. Participants were recruited by means of local advertisements. The participants were required to have caries-free maxillary and mandibular anterior teeth, without restorations on the labial surfaces. The central incisors had to be shade A2 or darker, judged by comparison with a valueoriented shade guide (VITA Classical, VITA Lumin, VITA Zahnfabrik, Bad Sackingen, Germany). Participants who had undergone previous tooth-whitening procedures; presented anterior restorations' were pregnant or lactating; and/or had severe internal tooth discoloration (eg, tetracycline stains, fluorosis, pulpless teeth), bruxism habits, or 
any other pathology that could cause sensitivity (eg, recession, dentin exposure) were excluded from the study because they would not be immediately eligible for a cosmetic treatment such as bleaching, given that the other restorative needs would need priority attention. Using the criteria described in the "Tooth Sensitivity (TS) Evaluation" section, the patients were asked about previous experiences with TS the week before the bleaching therapy began. Patients with TS equal to or greater than mild were also excluded from the study.

\section{Sample Size Calculation}

The primary outcome of this study was color changes in the participants' teeth, which were evaluated with a spectrophotometer (Vita Easyshade, Vident, Brea, CA, USA). Previous studies ${ }^{2-26}$ have reported that 2 bleaching sessions with the product Whiteness 35\% HP Maxx, a predecessor of Whiteness HP Automixx 35 (FGM Dental Products, Joinville, SC, Brazil), produced a whitening effect of about $8(\Delta \mathrm{E})$. To detect a difference of $3.5(\Delta \mathrm{E})$, which is clinically perceptible when comparing pairs of teeth per study group, with a power of $80 \%$ and an alpha of 5\%, a minimum sample size of 22 patients per group was required. The sample size was calculated on the website www.sealedenvelope.com. The present study was powered to detect a significantly high effect.

\section{Allocation Concealment, Experimental Groups, and Blinding}

Patients who met the inclusion criteria were asked about their daily cola-based soft drink (Coca-Cola, São Paulo, Brazil) consumption. Those who did not drink cola-based soft drinks were allocated to the control group. No other dietary restrictions were placed on the participants in the control group.

The patients who had reported drinking cola-based soft drinks at least twice a day every day were placed in the experimental group. No dietary restrictions were placed on the participants in the experimental group. Apart from their daily Coca-Cola intake ( 2 to 3 glasses daily), these patients were instructed to make mouth rinses with instant Coca-Cola $(50 \mathrm{~mL})$ for $30 \mathrm{~s}$, 4 times a day ( $8 \mathrm{am}, 12 \mathrm{am}, 4$ $\mathrm{pm}$, and $8 \mathrm{pm})$. They were instructed to perform one of the rinses immediately after each bleaching session while still in the office. For this procedure, participants received bottles of Coca-Cola, and were instructed to wait at least 15 mins after their Coca-Cola rinses before washing the mouth with clean water, brushing their teeth, or eating.
The aim of this procedure was to increase the exposure of bleached teeth to Coca-Cola. As a measure of adherence to the experimental protocol, participants were given a diary in which they were asked to take note of the number of CocaCola mouth rinses performed daily. They were instructed about the importance of the procedure and to report any time they forgot or were unable to perform mouth rinses. Participants and operators could not be blinded to the study groups. However, the evaluator who performed the color assessments was blinded to the treatments.

\section{Study Intervention}

All subjects received dental prophylaxis 2 weeks before the bleaching protocol began. The gingival tissue of the teeth to be bleached was isolated using a light-cured resin dam (Top Dam, FGM Dental Products). The bleaching procedure was performed using a 35\% HP gel (Whiteness HP Automixx 35, FGM Dental Products) according to the manufacturer's instructions (Table 1) for 2 sessions, three 15 min applications per session, with an interval of 7 days between each session. At this time, all participants were instructed to brush their teeth regularly using toothpaste without desensitizing or bleaching agents. Regarding oral hygiene, all participants were instructed to brush their teeth regularly and were asked to refrain from using whitening toothpaste and mouthwash containing peroxides.

\section{Color Evaluation}

Teeth color was recorded before the bleaching procedure for the baseline and 30 days after the end of the bleaching procedure. For this purpose, we used 1 objective (Vita Easyshade spectrophotometer, Vident) and 2 subjective instruments (value-oriented shade guides from VITA Classical and VITA Bleachedguide 3D, VITA Zahnfabrik). Color evaluation was completed in a room under artificial lightning conditions without interference from outside light. For all measurements, the color of the middle third of the central incisors was checked.

For the objective evaluation, a preliminary impression of the maxillary arch using high-putty silicone Coltoflax (Vigodent, Rio de Janeiro, RJ, Brazil) was taken, and a window on the labial surface of the silicon guide was created by a metal device with a $6-\mathrm{mm}$ radius to standardize the area for color evaluation with the spectrophotometer. The color was determined using the parameters of the digital spectrophotometer, on which the following values were indicated: $\mathrm{L}^{*} \mathrm{a}^{*}$ and $\mathrm{b}^{*}$ parameters were recorded. $\mathrm{L}^{*}$ represents the value from 0 (black) to 100 (white), and $\mathrm{a}^{*}$ and $\mathrm{b}^{*}$ represent 
Table I Product and Composition and Application Regimen

\begin{tabular}{|l|l|l|}
\hline Product & Composition* & Application Regimen* \\
\hline $\begin{array}{l}\text { Whiteness HP Automixx } 35 \\
\text { (FGM Dental Products, } \\
\text { Joinville, SC, Brazil) }\end{array}$ & $\begin{array}{l}\text { Hydrogen peroxide 35\%, thickener, } \\
\text { colorant, glycol, Inorganic load and } \\
\text { water. }\end{array}$ & $\begin{array}{l}\text { I. Insert the lip retractor; } \\
\text { 2. To apply the light-cured gingival barrier; } \\
\text { 3. Mix the two phases gel in pot in a ratio of 3: I drops peroxide to thickener; } \\
\text { 4. With the aid of a nozzle, spreading the gel on the buccal teeth, forming } \\
\text { a layer between } 0.5 \text { and I mm thick; } \\
\text { 5. Leave the gel acting for I5 mins and stir the product every } 5 \text { mins for the } \\
\text { release of bubbles; } \\
\text { 6. Aspire excess gel, wash your teeth and the application was repeated twice } \\
\text { more, applied } 3 \text { times, totaling } 45 \text { mins of contact with the teeth; } \\
\text { 7. Aspire excess gel, wash your teeth and remove the gingival barrier. }\end{array}$ \\
\hline
\end{tabular}

Note: *According to the manufacturer's instructions.

the shade, where $\mathrm{a}^{*}$ is the measurement along the red-green axis and $b^{*}$ is the measurement along the yellow-blue axis. The difference between baseline and each recall period $\left(\Delta \mathrm{E}^{*}\right)$ was calculated by using the following formula: $\Delta \mathrm{E}=\left[\left(\Delta \mathrm{L}^{*}\right)^{2}\right.$ $\left.+\left(\Delta \mathrm{a}^{*}\right)^{2}+\left(\Delta \mathrm{b}^{*}\right)^{2}\right] 1 / 2$. For the subjective evaluation, the 16 tabs of the shade guide (VITA classical, VITA Zahnfabrik) were arranged from lightest to darkest as follows: B1, A1, B2, D2, A2, C1, C2, D4, A3, D3, B3, A3.5, B4, C3, A4, C4. Color changes were calculated from the beginning of the active phase to the individual recall times by calculating the change in the number of shade guide units ( $\triangle \mathrm{SGUs})$, which occurred toward the lighter end of the value-oriented list of shade tabs. Two calibrated blind examiners participated in this evaluation, and in the event of disagreement between the examiners, a consensus was reached.

\section{Tooth Sensitivity Evaluation}

Patients were instructed to fill out a form to record their perception of TS immediately after, $1 \mathrm{hr}$ after, $24 \mathrm{hrs}$ after, and up to $48 \mathrm{hrs}$ after each session. The patients should fill out the form any time they felt pain. It was also explained to them that if they did not feel any TS, the intensity in the pain scales would be zero. In case of pain, the patients should report their TS intensity, using a 5-point numeric rating scale (NRS) using the following criteria: $0=$ none, $1=$ mild, 2 = moderate, $3=$ considerable, and $4=$ severe, and the visual analog scale (VAS). This scale employs a 10$\mathrm{cm}$ horizontal line with the words "no pain" at one end and "worst pain" at the opposite end.

The median (NRS) and the average (VAS) of bleaching-induced TS intensity as experienced by each patient were calculated throughout the bleaching treatment period. The overall percentage of patients with TS, as well the total number of days on which patients experienced TS, was also evaluated.

\section{Statistical Analysis}

The analysis followed the intent-to-treat protocol and involved all participants who were randomly assigned in their respective groups (Figure 1). The median and interquartile range of color change in $\triangle \mathrm{SGU}$, as well as the means and standard deviations of color change in $\Delta \mathrm{E}$ between baseline and 30 days after bleaching, were calculated. To assess whether the bleaching therapies were effective, data from both groups were compared using the Mann-Whitney test for the $\Delta \mathrm{SGU}$ data and the Student's $t$-test for $\Delta \mathrm{E}$.

The absolute risks of bleaching-induced TS for both groups were compared by means of the Chi-square test ( $\alpha=0.05$, test for proportion of dependent data ratio). The relative risk and the confidence interval $(\mathrm{CI})$ for the effect size were also calculated.

The bleaching-induced TS intensity data set for both the VAS and NRS scales were plotted in histograms and inspected for normal distributions. The VAS data were considered to have a normal distribution, and the groups were compared using a Student's $t$-test. Otherwise, because the NRS data did not have a normal distribution, the groups were compared using the sign test and Wilcoxon signedrank test $(\alpha=0.05)$.

\section{Results}

A total of 58 participants were examined according to the eligibility criteria (Figure 1), but only 44 participants remained for the clinical trial. The main reasons for the exclusions were shade (incisors lighter than A2) and the presence of anterior restorations. The mean age (years) of the participants and the 

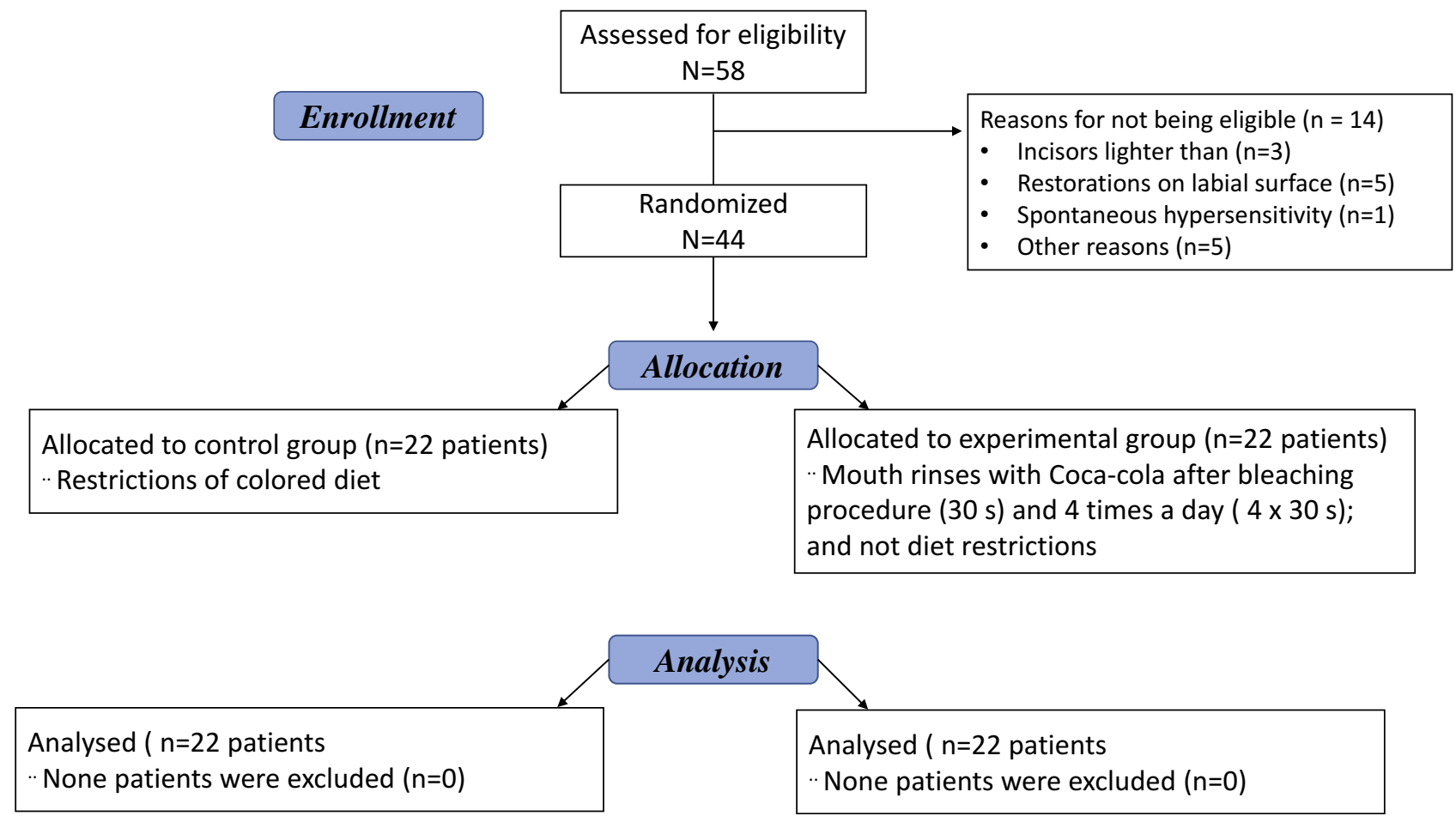

Figure I Flow diagram of the clinical trial, including detailed information on the participants.

baseline SGUs are described in Table 2. One can observe comparable data among treatment groups.

\section{Adherence to Protocol and Loss to Follow-Up}

All participants made mouth rinses with $50 \mathrm{~mL}$ of CocaCola for $30 \mathrm{~s}, 4$ times of day and of 44 participants, 4 forgot to do the mouth rinse at 8 am 1 day. None of the patients discontinued the intervention, and all participants attended the recall visits during the bleaching treatment. Figure 1 depicts the flow diagram with detailed information on the participants in the different phases of the study design.

\section{Color Change}

After 30 days, a significant whitening effect was detected by the three colors measurement tools for both the evaluated groups (Table 3). However, no statistically significant

Table 2 Baseline Characteristics of the Participants Included in This Clinical Trial, for Both the Study Groups

\begin{tabular}{|l|l|l|}
\hline Characteristics & Control & Experimental \\
\hline Age (average \pm SD, years) & $25.6 \pm 4.1$ & $23.6 \pm 3.7$ \\
Baseline color (average \pm SD, SGU VC) & $6.95 \pm 3.6$ & $6.09 \pm 3.2$ \\
\hline
\end{tabular}

Abbreviations: SGU VC, Shade Guide Unit/Vita Classical guide, SD, standard deviation. difference was observed between the study groups (Table 3; $\mathrm{p}>0.08)$.

\section{Tooth Sensitivity}

The absolute risks of bleaching-induced TS were 32\% (control group; 95\% CI 16-52) and 27\% (experimental group; 95\% CI 13-48), and there was no significant difference between the 2 groups (risk ratio: 0.8 [95\% IC 0.34-2.14] $\mathrm{p}=0.74$; Figure 2). Regarding bleaching-induced TS intensity, the present results showed higher, significant scores in the VAS and NRS values after $1 \mathrm{hr}$ of bleaching ( $<<0.05$; Table 4). However, bleaching-induced TS intensity decreased in the post-treatment period between 1 and $24 \mathrm{hrs}$, similar to values obtained during treatment.

Table 3 Means and Standard Deviations of the Color Measured by Vita Classical and Vita Bleached Guide ( $\Delta S G U)$ and by Easyshade Spectrophotometer $(\Delta \mathrm{E})$ Between Baseline versus 30 Days After Bleaching for Two Study Groups*

\begin{tabular}{|l|l|l|l|}
\hline Color Evaluation & Control & Experimental & p-value \\
\hline$\Delta$ SGU (Vita Classical) & $5.1 \pm 2.9$ & $4.9 \pm 2.7$ & $0.70^{*}$ \\
$\Delta \mathrm{SGU}$ (Vita Bleachedguide 3D) & $6.5 \pm 3.0$ & $5.7 \pm 3.2$ & $0.80^{*}$ \\
$\Delta \mathrm{E}$ & $8.9 \pm 2.4$ & $9.0 \pm 3.6$ & $0.08^{* *}$ \\
\hline
\end{tabular}

Notes: *Student's t-test $(\alpha=0.05)$; **Mann-Whitney test $(\alpha=0.05)$. 


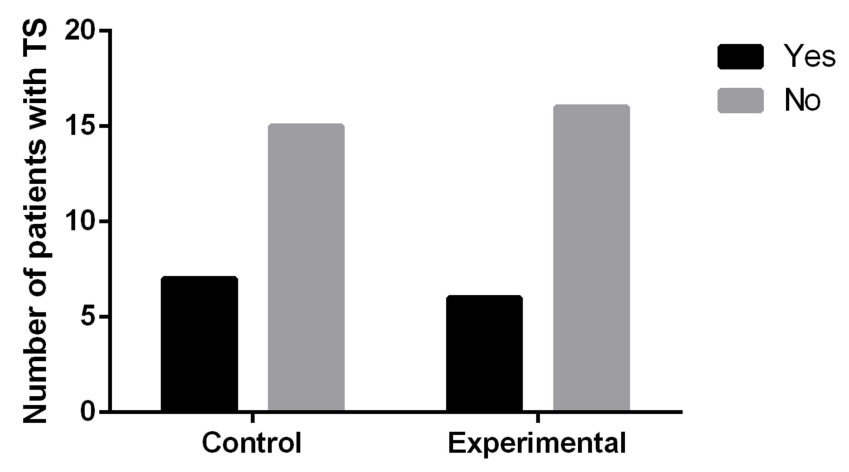

Figure 2 Comparison of the number of patients who experienced tooth sensitivity (TS) at least once during the bleaching treatment in both the study groups.

A significant reduction in bleaching-induced TS was observed until $48 \mathrm{hrs}$ for both scales ( $\mathrm{p}>0.05$; Table 4).

When both groups were compared, higher, significant scores in the VAS ( 1 to $24 \mathrm{hrs}$ ) and in the NRS ( $1 \mathrm{hr}$ after and 1 to $24 \mathrm{hrs}$ ) were observed for the experimental group in comparison with the control group ( $p<0.05$; Table 4).

\section{Discussion}

Patients are often instructed by their dentists to stay on a white diet during active bleaching treatment and to refrain from smoking, as well as drinking coffee, red wine, and colored soft drinks. All these directions are based on previous in vitro studies that reported that staining susceptibility increased when the bleached enamel was exposed to colored drinks such as red wine, coffee and cola-based soft drink, especially after the bleaching treatment. ${ }^{8-11}$ Thus, the alterations promoted by the bleaching agents in the enamel surface could favor greater retention of coloring agents.

However, regarding the change of color, the mouth rinses with Coca-Cola did not affect the effectiveness of tooth bleaching in comparison with the control group. Both the protocols demonstrated significant whitening after 2 bleaching sessions. Thus, the results oppose the idea that acidic beverages containing coloring agents, as cola-based soft drinks, that cause extrinsic stains reduce bleaching potential ${ }^{11-13}$ and it lead us to accept our first null hypothesis.

It is worth mentioning that this study associated objective and subjective methods of color assessment. Although the common sense that, spectrophotometer gives more accurate results, ${ }^{24}$ this instrument is yet not currently used in clinical practice. On the other hand, shade guide units are the most used tools for color evaluation in the clinicians' armamentarium. ${ }^{24}$ Also, recently Pecho et al $(2016)^{27}$ indicate that instrumental shade determination should be accompanied by experienced human visual assessment to better visual perception of color change. This is probably, the main reason that both methods, subjective and objective are frequently used in clinical trial evaluating bleaching materials and techniques, ${ }^{16,24,28,29}$ as well as, in the present study.

In agreement with the results obtained in this study, Rezende et al $2013^{16}$ and De Geus et al $2015^{28}$ clinically demonstrated that exposure to coffee or smoking, respectively, during at-home bleaching with 10-16\% carbamide peroxide did not affect the effectiveness of dental bleaching. ${ }^{16,28}$ Additionally, the role of a white diet, with restrictions on coffee, tea, red wine, and dark fruits, was not significantly associated with higher bleaching effectiveness. ${ }^{14}$ These studies did not evaluate the effect of cola-based soft drinks, which have a lower $\mathrm{pH}$ than coffee, tea, and red wine. ${ }^{11,13,28}$ In fact, it was previously demonstrated that cola-based soft drinks stain bleached enamel similar or even more than coffee, possibly because a lower $\mathrm{pH}$ increases the porosity ${ }^{11}$ provided by the potential of demineralization on the tooth surface ${ }^{11,13}$ and increases the retention of coloring agents.

However, the experimental conditions tested in existing in vitro studies varied greatly in, for instance, the time of

Table 4 Intensity of Tooth Sensitivity in VAS (Average \pm SD) and NRS (Median/Interquartile Range) in Several Evaluation Times, as Well as, Statistical Evaluation

\begin{tabular}{|l|l|l|l|l|}
\hline \multirow{2}{*}{ Evaluation Time } & \multicolumn{2}{l|}{ VAS $(*)$} & \multicolumn{2}{l|}{ NRS $(* *)$} \\
\cline { 2 - 5 } & Control & Experimental & Control & \multicolumn{2}{l|}{ Experimental } \\
\hline During treatment & $1.5 \pm 2.6 \mathrm{aA}$ & $1.6 \pm 2.4 \mathrm{aD}$ & $0(0 / \mathrm{l})^{\mathrm{a}, \mathrm{A}}$ & $0.5(0 / 2)^{\mathrm{a}, \mathrm{C}}$ \\
I h after & $3.1 \pm 3.2 \mathrm{bB}$ & $3.8 \pm 3.7 \mathrm{bE}$ & $1.5(0 / 2.75)^{\mathrm{b}, \mathrm{B}}$ & $2.5(0.25 / 2.75)^{\mathrm{c}, \mathrm{D}}$ \\
I to $24 \mathrm{hrs}$ & $\mathrm{l}$ & $2.1 \pm 2.5 \mathrm{cA}$ & $0(0 / 0)^{\mathrm{d}, \mathrm{A}}$ & $0(0 / \mathrm{I})^{\mathrm{e}, \mathrm{C}}$ \\
Until $48 \mathrm{hrs}$ & $0.4 \pm 1.7 \mathrm{eC}$ & $0.5 \pm 2.1 \mathrm{eF}$ & $0(0 / 0)^{\mathrm{f}, \mathrm{A}}$ & $0(0 / 0)^{\mathrm{f}, \mathrm{C}}$ \\
\hline
\end{tabular}

Notes: *VAS (Student's $t$-test for independent samples for comparison between groups in each evaluation time; different lowercase letters means groups statistically different; $p<0.05$ and; $t$-test for dependent samples for comparison between evaluation time for each group; different uppercase letters means groups statistically different; $P<0.05)$. $* * N R S$ (Sign test for independent samples for comparison between groups in each evaluation time; different lowercase superscript letters means groups statistically different; $p<0.05$ and Wilcoxon test for comparison between evaluation time for each group; different uppercase superscript letters means groups statistically different; $\mathrm{p}<0.05$ ). 
exposure to coloring agents. ${ }^{9,11,30}$ Because of this, they were unable to simulate a clinical condition. For example, in Pirollo et al $2014^{11}$ after the bleaching procedure $(35 \%$ HP), exposure to a cola-based soft drink increased from 10 mins to $72 \mathrm{hrs}$. In the present study, the time of bleached tooth exposure to Coca-Cola was determined by considering the time it took to swallow liquid. ${ }^{31}$ Considering that this probably did not take longer than 2 to $5 \mathrm{~s}, 4$ daily mouth rinses performed for $30 \mathrm{~s}$ each would represent excessive intake of the beverage. Furthermore, clinical situations that imitate real-life conditions, such as patients brushing their teeth normally and human saliva-replacing materials lost by the tooth structure during bleaching ${ }^{32,33,34}$ could attempt to reproduce laboratory setting, but they certainly will not able to completely simulate the cascade of events of the oral environment, as in a clinical trial.

Another aspect related to color, which is worthwhile to explore, is that substances able to stain extrinsically, such as cola-based soft drink, ${ }^{1-13}$ are compounds constituted of chromogenic polyphenols. These compounds are believed to bind to proteins, such as the pellicle or bacteria on the surface of the teeth. ${ }^{31}$ Actually, these macromolecular chains practically incapable of passing through human enamel, which acts as a semipermeable membrane that allows the passage of low molecular weight molecules. ${ }^{35}$

Moreover, it is known that the bleaching process results from oxidation of the organic tissues of human dentin, and not of the enamel structure. ${ }^{36}$ Furthermore, the extrinsic stains by adsorbed pigments and biofilm are prone to be efficiently removed by professional dental prophylaxis. In this study, after 30 days of the bleaching procedure, maintenance of the effectiveness of bleaching was observed during the patient follow-ups.

In terms of TS, when it was compared to the absolute risk of sensitivity, no significant difference was observed between the study groups. The literature hypothesizes several factors that can be associated with TS, among them the $\mathrm{pH}$ of bleaching gels, ${ }^{26}$ dentin thickness, ${ }^{37}$ and the concentration of bleaching agents. ${ }^{38}$ However, even though the current study evaluated the presence and absence of Coca-Cola mouth rinses, the bleaching agent and protocol were the same for both study groups. Doing it like this helps us to understand the similar risk of sensitivity, leading to reject the second null hypothesis.

Although the mechanism responsible for the bleachinginduced TS is unclear, it is hypothesized that, during bleaching treatment, the HP increases the permeability of the enamel and diffuses through the dentin, reaching the pulp chamber. ${ }^{25,26}$ Thus, chemical products resulting from the degradation of HP penetrate the pulp chamber ${ }^{23}$ and lead to the activation of nociceptive sensors and transient inflammatory reactions with higher intensity after the procedure, ${ }^{25,39,40}$ but this is usually reduced within $48 \mathrm{hrs}^{41}$ In fact, it could explain the significantly higher bleaching-induced TS scores after $1 \mathrm{hr}$ of bleaching, followed by a continuous reduction in these values to up $48 \mathrm{hrs}$, for both the study groups.

Although the exposure to Coca-Cola did not affect bleaching effectiveness, higher and significant scores for bleaching-induced TS intensity were detected in comparison to patients who did not drink Coca-Cola, leading to reject the third null hypothesis. In contrast to Rezende et al $2013,{ }^{16}$ whose findings found that coffee did not affect TS, it should be considered that Coca-Cola has a more acidic $\mathrm{pH}(\cong 2.53)$ than coffee $(\cong 4.46){ }^{11-13,42}$ A well-known fact in the literature is that the erosive potential of Coca-Cola affects not only mineral loss and dental enamel ${ }^{19,43}$ but also, in epidemiological and clinical evidences, calcium homeostasis and bone turnover ${ }^{19,44}$ even in a short time period. ${ }^{19}$ It is worth mentioning that, although the evaluation of the erosive potential was not within the scope of the present study, dental erosion was not observed during the follow-ups of this study, especially for patients who ingested Coca-Cola.

During the bleaching process, the permeability of the enamel surface with HP diffusing through the dentin had increased. ${ }^{44}$ This idea that the bleaching process occurs in organic tissues ${ }^{36}$ has been reaffirmed by recent studies that suggested that the HP bleaches the dentin by oxidizing dentin phosphoproteins (the main noncollagenous protein in dentin), ${ }^{15,45}$ may be due to the denaturation of collagen and its effects on amide I and III in dentin. ${ }^{15}$ Certainly, a reduction in the amount of minerals in enamel also promoted the exposure of acid and carbonated beverages such as Coca-Cola and could intensify the diffusion of HP through dentin, especially given that the HP is more readily dispersed within deeper regions than the outer portions due to a reduction in mineral content and density ${ }^{6,45}$ and, probably, would activate more nociceptive sensors with inflammatory reactions in dental pulp.

Actually, interesting studies aiming to find associated factors for dental hypersensitivity demonstrated a positive trend between TS and dietary acid intake. ${ }^{21,46}$ O'Toole and Bartlett $^{21}$ demonstrated that dental hypersensitivity is common among patients who consumed acidic drinks 2 or more times a day, as well as acidic drinks between meals without 
considering the erosive tooth wear, as occurred in the current study. Although the dental hypersensitivity and bleaching-induced tooth sensitivity cannot be confused. ${ }^{23}$ These previous findings help confirm our hypothesis that CocaCola in mouth rinses is a potential factor for the increase in TS intensity.

Although only the Coca-Cola was evaluated and the commercial products show particularities not completely clear in the composition hindering an extrapolation, the consumption of other carbonated and acidic beverages should seem with caution, since that it was related to be one of the potential factors for the sensitivity in this study. Also, it is worth mentioning the limitations of the experimental design of this study. Unfortunately, the participants were not randomized to the study groups because this procedure means that all patients have the same chance to be allocated in any group. As in one of the groups, the patients need to intake routinely Coca-Cola, it would not be ethically correct submitting patients which do not intake routinely Coca-Cola to intake 2 to 3 glasses daily during 2 weeks, as well as, described in previous clinical trials evaluating the effect of coffee or smoking during in bleaching treatment. ${ }^{16,28}$ In spite of this, these findings are stronger than those obtained in laboratory conditions. In addition, dietary restrictions were only utilized in the control group. If differences were detected between groups, we would not be able to discriminate between the effects of Coca-Cola exposure and those of other staining foods in the patient's diet. Another limitation of the study is that the participants were predominantly young people with good oral hygiene, without restorations. These results may not be applicable to the elderly population and those with poor oral hygiene conditions.

Future clinical trials are needed to better evaluate the influence of other components of the diet in whitening effectiveness and TS associated to bleaching therapies, especially in the elderly populations.

\section{Conclusion}

Although the exposure to cola-based soft drinks during the in-office bleaching treatment did not affect the effectiveness of bleaching, a higher intensity of tooth sensitivity was related by patients who ingested cola-based soft drinks during the bleaching period. This means that the exposition to cola-based soft drinks should be avoided, mainly when higher concentrated hydrogen peroxide, as indicated for in-office bleaching treatment was used.

\section{Data Sharing Statement}

According to polices of the journal, data sharing statement:

1. The authors intend to share individual deidentified participant data;

2. The raw data obtained in the research will be available. Data related to color change and sensitivity. No personal data will be available from survey participants;

3. Ethics committee approval documents and clinical research database records will be available;

4. The data will be available in repository, through the link on the university web page;

5. Data will be available from publication for an indefinite period.

\section{Disclosure}

The authors report no conflicts of interest in this work.

\section{References}

1. Samorodnitzky-Naveh GR, Geiger SB, Levin L. Patients' satisfaction with dental esthetics. J Am Dent Assoc. 2007;138(6):805-808. doi:10.14219/jada.archive.2007.0269

2. Dahl JE, Pallesen U. Tooth bleaching - a critical review of the biological aspects. Crit Rev Oral Biol Med. 2003;14(4):292-304. doi: $10.1177 / 154411130301400406$

3. Joiner A. The bleaching of teeth: a review of the literature. $J$ Dent. 2006;34(7):412-419. doi:10.1016/j.jdent.2006.02.002

4. Gurgan S, Cakir FY, Yazici E. Different light-activated in-office bleaching systems: a clinical evaluation. Lasers Med Sci. 2010;25 (6):817-822. doi:10.1007/s10103-009-0688-x

5. Hosoya N, Honda K, Iino F, Arai T. Changes in enamel surface roughness and adhesion of streptococcus mutans to enamel after vital bleaching. J Dent. 2003;31(8):543-548. doi:10.1016/S0300-5712(03)00109-X

6. Tanaka R, Shibata Y, Manabe A, Miyazaki T. Micro-structural integrity of dental enamel subjected to two tooth whitening regimes. Arch Oral Biol. 2010;55(4):300-308. doi:10.1016/j.archoralbio.2010.02.009

7. Schiavoni RJ, Turssi CP, Rodrigues AL, Serra MC, Pécora JD, Fröner IC. Effect of bleaching agents on enamel permeability. $\mathrm{Am}$ J Dent. 2006;19(5):313-316.

8. Berger SB, Coelho AS, Oliveira VAP, Cavalli V, Giannini M. Enamel susceptibility to red wine staining after $35 \%$ hydrogen peroxide bleaching. J Appl Oral Sci. 2008;16(3):201-204. doi:10.1590/ s1678-77572008000300007

9. Liporoni PCS, Souto CMC, Pazinatto RB, et al. Enamel susceptibility to coffee and red wine staining at different intervals elapsed from bleaching: a photoreflectance spectrophotometry analysis. Photomed Laser Surg. 2010;28(S2):S-105-S-109. doi:10.1089/pho.2009.2627

10. Attia ML, Aguiar FHB, Mathias P, Ambrosano GMB, Fontes CM, Liporoni PCS. The effect of coffee solution on tooth color during home bleaching applications. Am J Dent. 2009;22(3):175-179.

11. Pirolo R, Mondelli RFL, Correr GM, Gonzaga CC, Furuse AY. Effect of coffee and a cola-based soft drink on the color stability of bleached bovine incisors considering the time elapsed after bleaching. $J \mathrm{Appl}$ Oral Sci. 2014;22(6):534-540. doi:10.1590/1678-775720130578

12. da Silva TM, da Silva NY, Gonçalves LL, Alves LP, Fernandes AU, Gonçalves SEP. Staining beverages and cigarette smoke on composite resin and human tooth fluorescence by direct spectrometry. J Contemp Dent Pract. 2017;18(5):352-357. doi:10.5005/jp-journals-10024-2045 
13. Farawati FAL, Hsu SM, O’Neill E, Neal D, Clark A, EsquivelUpshaw J. Effect of carbamide peroxide bleaching on enamel characteristics and susceptibility to further discoloration. J Prosthet Dent. 2019;121(2):340-346. doi:10.1016/j.prosdent.2018.03.006

14. Matis B, Wang G, Matis J, Cook N, Eckert G. White diet: is it necessary during tooth whitening? Oper Dent. 2015;40(3):235-240. doi:10.2341/14-019-LIT

15. Redha O, Strange A, Maeva A, et al. Impact of carbamide peroxide whitening agent on dentinal collagen. J Dent Res. 2019;98 (4):443-449. doi:10.1177/0022034518822826

16. Rezende M, Loguercio A, Reis A, Kossatz S. Clinical effects of exposure to coffee during at-home vital bleaching. Oper Dent. 2013;38(6):E229-36. doi:10.2341/12-188-c

17. Bere E, Sørli Glomnes E, Te Velde SJ, Klepp KI. Determinants of adolescents' soft drink consumption. Public Health Nutr. 2008;11 (1):49-56. doi:10.1017/s1368980007000122

18. Popkin BM, Nielsen SJ. The sweetening of the world's diet. Obes Res. 2003;11(11):1325-1332. doi:10.1038/oby.2003.179

19. Kristensen M, Jensen M, Kudsk J, Henriksen M, Mølgaard C. Shortterm effects on bone turnover of replacing milk with cola beverages: a 10-day interventional study in young men. Osteoporos Int. 2005;16 (12):1803-1808. doi:10.1007/s00198-005-1935-z

20. Denova-Gutiérrez E, Méndez-Sánchez L, Muñoz-Aguirre $P$, Tucker KL, Clark P. Dietary patterns, bone mineral density, and risk of fractures: a systematic review and meta-analysis. Nutrients. 2018;10(12):1922. doi:10.3390/nu10121922

21. O'Toole S, Bartlett D. The relationship between dentine hypersensitivity, dietary acid intake and erosive tooth wear. $J$ Dent. 2017;67:84-87. doi:10.1016/j.jdent.2017.10.002

22. Haywood VB. Treating sensitivity during tooth whitening. Compend Contin Educ Dent. 2005;26(9 Suppl 3):11-20.

23. Kielbassa AM, Maier M, Gieren AK, Eliav E. Tooth sensitivity during and after vital tooth bleaching: a systematic review on an unsolved problem. Quintessence Int. 2015;46(10):881-897. doi:10.3290/j.qi.a34700

24. Mena-Serrano AP, Garcia E, Luque-Martinez I, Grande R, Loguercio AD, Reis A. A single-blind randomized trial about the effect of hydrogen peroxide concentration on light-activated bleaching. Oper Dent. 2016;41(5):455-464. (). doi:10.2341/15-077-C

25. Reis A, Kossatz S, Martins GC, Loguercio AD. Efficacy of and effect on tooth sensitivity of in-office bleaching gel concentrations: a randomized clinical trial. Oper Dent. 2013;38(4):386-393. doi:10.2341/12-140-c

26. Tay LY, Kose C, Loguercio AD, Reis A. Assessing the effect of a desensitizing agent used before in-office tooth bleaching. J Am Dent Assoc. 2009;140(10):1245-1251. doi:10.14219/jada.archive.2009.0047

27. Pecho OE, Ghinea R, Alessandretti R, Pérez MM, Della Bona A. Visual and instrumental shade matching using CIELAB and CIEDE2000 color difference formulas. Dent Mater. 2016;32 (1):82-92. doi:10.1016/j.dental.2015.10.015

28. De Geus JL, Bersezio C, Urrutia J, et al. Effectiveness of and tooth sensitivity with at-home bleaching in smokers: a multicenter clinical trial. J Am Dent Assoc. 2015;146(4):233-240. doi:10.1016/ j.adaj.2014.12.014

29. Martins I, Onofre S, Franco N, et al. Effectiveness of in-office hydrogen peroxide with two different protocols: a two-center randomized clinical trial. Oper Dent. 2018;43(4):353-361. doi:10.2341/17$128-\mathrm{c}$
30. Azer SS, Hague AL, Johnston WM. Effect of $\mathrm{pH}$ on tooth discoloration from food colorant in vitro. J Dent. 2010;38(SUPPL. 2):e106-9. doi:10.1016/j.jdent.2010.07.014

31. Addy M, Moran J. Mechanisms of stain formation on teeth, in particular associated with metal ions and antiseptics. Adv Dent Res. 1995;9:450-456. doi:10.1177/08959374950090041601

32. Santos RS, Filho EDM. Sonar doppler as an instrument of deglutition evaluation International. Arch Otorhinolaryngol. 2006;10(3):182-191.

33. Sa Y, Sun L, Wang Z, et al. Effects of two in-office bleaching agents with different ph on the structure of human enamel: an in situ and in vitro study. Oper Dent. 2012;38(1):100-110. doi:10.2341/11-173-1

34. Justino LM, Tames DR, Demarco FF. In situ and in vitro effects of bleaching with carbamide peroxide on human enamel. Oper Dent. 2004;29(2):219-225.

35. Nicholson JW. Biologic considerations. In: Summitt JB, Robbins JW, Hilton TJ, Schwartz RS, dos Santos J editors. Fundamentals of Operative Dentistry: A Contemporary Approach ed 3. Chicago: Quintessence Publishing, 2006:1-36.

36. Kawamoto K, Tsujimoto Y. Effects of the hydroxyl radical and hydrogen peroxide on tooth bleaching. J Endod. 2004;30(1):45-50. doi:10.1097/00004770-200401000-00010

37. Rezende M, Loguercio AD, Kossatz S, Reis A. Predictive factors on the efficacy and risk/intensity of tooth sensitivity of dental bleaching: a multi regression and logistic analysis. J Dent. 2016;45:1-6. doi:10.1016/j.jdent.2015.11.003

38. Soares DG, Basso FG, EC V P, Garcia LDFR, Hebling J, De Souza Costa CA. Effective tooth-bleaching protocols capable of reducing H2O 2 diffusion through enamel and dentine. J Dent. 2014;42 (3):351-358. doi:10.1016/j.jdent.2013.09.001

39. Caviedes-Bucheli J, Ariza-García G, Restrepo-Méndez S, RíosOsorio N, Lombana N, Muñoz HR. The effect of tooth bleaching on substance P expression in human dental pulp. J Endod. 2008;34 (12):1462-1465. doi:10.1016/j.joen.2008.09.013

40. Costa CAS, Riehl H, Kina JF, Sacono NT, Hebling J. Human pulp responses to in-office tooth bleaching. J Esthet Restor Dent. 2014;26 (5):356. doi:10.1016/j.tripleo.2009.12.002

41. Rezende M, Coppla F, Chemin K, Chibinski A, Loguercio A, Reis A. Tooth sensitivity after dental bleaching with a desensitizer-containing and a desensitizer-free bleaching gel: a systematic review and meta-analysis. Oper Dent. 2019;44(2):E58-74. doi:10.2341/17-253-1

42. Jameel RA, Khan SS, Rahim ZHA, Bakri MM, Siddiqui S. Analysis of dental erosion induced by different beverages and validity of equipment for identifying early dental erosion, in vitro study. $J$ Pak Med Assoc. 2016;66(7):843-848.

43. Lussi A, Carvalho TS. Analyses of the erosive effect of dietary substances and medications on deciduous teeth. PLoS One. 2015;10 (12):1-15. doi:10.1371/journal.pone.0143957

44. Xavier A, Rai K, Hegde A, Shetty S, Spectroscopic A. Surface microhardness study on enamel exposed to beverages supplemented with lower iron concentrations. J Clin Pediatr Dent. 2015;39 (2):161-167. doi:10.17796/jcpd.39.2g52v661835527526

45. Jiang T, Guo YR, Feng XW, et al. Hydrogen peroxide might bleach natural dentin by oxidizing phosphoprotein. J Dent Res. 2018;97 (12):1339-1345. doi:10.1177/0022034518784260

46. West NX, Sanz M, Lussi A, Bartlett D, Bouchard P, Bourgeois D. Prevalence of dentine hypersensitivity and study of associated factors: a European population-based cross-sectional study. J Dent. 2013;41(10):841-851. doi:10.1016/j.jdent.2013.07.017 


\section{Publish your work in this journal}

Clinical, Cosmetic and Investigational Dentistry is an international, peer-reviewed, open access, online journal focusing on the latest clinical and experimental research in dentistry with specific emphasis on cosmetic interventions. Innovative developments in dental materials, techniques and devices that improve outcomes and patient satisfaction and preference will be highlighted. The manuscript management system is completely online and includes a very quick and fair peer-review system, which is all easy to use. Visit http://www.dovepress.com/testimonials.php to read real quotes from published authors.

Submit your manuscript here: https://www.dovepress.com/clinical-cosmetic-and-investigational-dentistry-journal 